\title{
DISLOCATION SUBSTRUCTURES OF INTERSTITIAL-FREE STEEL SUBJECTED TO LOW CYCLE FATIGUE AT VARIOUS STRAIN AMPLITUDE
}

Shih-Wei Mao

Department of Mechanical Engineering, R.O.C. Military Academy, Kaohsiung County, Taiwan, R.O.C., swmao1@gmail.com

Wen-Chun Lo

Department of Materials Science and Engineering, National Sun Yat-Sen University, Kaohsiung, Taiwan, R.O.C.

Hsing-Lu Huang

Department of Mechanical Engineering, R.O.C. Military Academy, Kaohsiung County, Taiwan, R.O.C.

New-Jin Ho

Department of Materials Science and Engineering, National Sun Yat-Sen University, Kaohsiung, Taiwan, R.O.C.

Follow this and additional works at: https://jmstt.ntou.edu.tw/journal

Part of the Mechanical Engineering Commons

\section{Recommended Citation}

Mao, Shih-Wei; Lo, Wen-Chun; Huang, Hsing-Lu; and Ho, New-Jin (2011) "DISLOCATION SUBSTRUCTURES OF INTERSTITIAL-FREE STEEL SUBJECTED TO LOW CYCLE FATIGUE AT VARIOUS STRAIN AMPLITUDE," Journal of Marine Science and Technology. Vol. 19: Iss. 2, Article 1.

DOI: $10.51400 / 2709-6998.2144$

Available at: https://jmstt.ntou.edu.tw/journal/vol19/iss2/1

This Research Article is brought to you for free and open access by Journal of Marine Science and Technology. It has been accepted for inclusion in Journal of Marine Science and Technology by an authorized editor of Journal of Marine Science and Technology. 


\title{
DISLOCATION SUBSTRUCTURES OF INTERSTITIAL-FREE STEEL SUBJECTED TO LOW CYCLE FATIGUE AT VARIOUS STRAIN AMPLITUDE
}

\author{
Shih-Wei Mao*, Wen-Chun Lo**, Hsing-Lu Huang*, and New-Jin Ho**
}

Key words: IF steel, dislocation, fatigue, grain size.

\begin{abstract}
The aim of this wok is to study the fatigue properties in relation to the microstructures of interstitial-free (IF) steel, a representative of body center cubic (BCC) materials, subjected to low cycle periodic stress. In order to compare the grain size effect on dislocation development, we used samples of two distinctive grain sizes, $70 \mu \mathrm{m}$ and $210 \mu \mathrm{m}$, prepared by different thermo-mechanical processes. We found (1) there was an initial softening when the stain amplitude was lower than $1.6 \%$ and the microstructure of the fatigued samples are cellular independent of the grain sizes and the amplitude of strain. (2) A re-entrant hardening following an initial softening stage took place for strain amplitude range from $0.2 \%$ to $0.4 \%$ in the samples of $70 \mu \mathrm{m}$ grains and $0.4 \%$ to $0.8 \%$ for those of 210 $\mu \mathrm{m}$ grains. Small cellular structures formed within a larger uncondensed cells structure at an initial stage. (3) While the mechanical behaviors of fatigue for both grain sizes are similar, differences do exist and are determined by strain localization as well as the multiple slip systems which can be triggered into active operation
\end{abstract}

\section{INTRODUCTION}

Dislocation structures can be divided into three types according to how the dislocations evolve when a relevant material is subject to periodic stress. The first dislocation often has a wavy type, for instance, in low cycle fatigued polycrystalline copper [12]. The second type is planar; frequently found in fatigued brass [3] and Fe-Al-Mn-C alloys [2]. A third type composed of a mixture of the above types exists in materials

Paper Submitted 10/03/08; revised 10/05/09; accepted 10/06/09. Author for correspondence: Shih-Wei Mao (e-mail:swmao1@gmail.com).

*Department of Mechanical Engineering, R.O.C. Military Academy, Kaohsiung County, Taiwan, R.O.C.

**Department of Materials Science and Engineering, National Sun Yat-Sen University, Kaohsiung, Taiwan, R.O.C. such as AISI 316 stainless steel $[4,5]$. Regardless of the dislocation evolution, these well studied materials share the common face center cubic structure (FCC) atomic arrangement. In contrast, seldom can one find similar reports on materials with a body center cubic (BCC) structure.

This is incentive to understand $\mathrm{BCC}$ materials because the slip systems are quite different from those in their FCC counterparts. The related dislocation evolutions are also expected to be different [8-11]. In FCC metals, dislocation interactions dictate the dislocation evolution. For BCC materials, the dislocation dynamics are generally dominated by the friction force between screw dislocations at low temperatures (about 0.1-0.2 $\mathrm{T}_{\mathrm{m}}, \mathrm{T}_{\mathrm{m}}$ : melting point) while activated by both friction and dislocation interactions at high temperatures. In addition, in BCC materials dislocation depends much on the relative slips of the screw and edge dislocation [8].

According to Mughrabi et al. [8,9], the mechanical behaviors as manifested in the S-N curves are affected by the carbon content, and the sizes of the associated dislocation cells are also affected by the applied strain amplitudes. Smaller cells with higher dislocation densities or larger cells with lower dislocation densities were found in tests conducted on both the FCC and BCC materials subjected to low strain amplitudes. This is largely because the dislocation structures evolve along similar paths. The cell size and dislocation density depend on how the dislocations interact, as Mughrabi $[8,9]$ pointed out, the dislocation evolution is determined by the relative slips in the screw and edge dislocations. We thus believe a specific investigation on BCC metals, in particular with various grain sizes, would help shed some light on the dislocation evolution and the fatigue properties in general.

\section{EXPERIMENTAL PROCEDURES}

The chemical composition of the IF steel used in this experiment is shown in Table 1.

In order to prepare samples with different grain sizes, a portion of the IF steel was annealed in the air at $1050^{\circ} \mathrm{C}$ for 10 minutes, while the other part at $1200^{\circ} \mathrm{C}$ for 120 minutes, all before being quenched into water. The resulting averaged 
Table 1. The elements of composition in IF steel.

\begin{tabular}{|c|c|c|c|c|c|c|c|c|}
\hline Elements & $\mathrm{C}$ & $\mathrm{Si}$ & $\mathrm{Mn}$ & $\mathrm{P}$ & $\mathrm{S}$ & $\mathrm{Ni}$ & $\mathrm{Cr}$ & $\mathrm{Fe}$ \\
\hline PPM & 50 & 110 & 1300 & 100 & 60 & 190 & 90 & Bal. \\
\hline
\end{tabular}

grain sizes for these specimens were about $70 \mu \mathrm{m}$ and $210 \mu \mathrm{m}$, respectively, for the two temperatures. Specimens were cut along the rolling direction, following the ASTM E647 protocol. The hour-glass shaped specimens had an outer diameter of $12 \mathrm{~mm}$, a gauge length of $17 \mathrm{~mm}$, and a gauge length diameter of $6 \mathrm{~mm}$. During the experiment, low cycle fatigue was completed using a computerized Instron 1332 hydraulic testing machine performed at $\mathrm{R}=-1$ (strain ratio, $\mathrm{R}=\varepsilon_{\min } / \varepsilon_{\max }$ ) under a strain rate of $1 \times 10^{-3} \mathrm{~s}^{-1}$.

After the low cycle fatigue test was completed, the specimens were sliced perpendicular to the loading axis into $0.6 \mathrm{~mm}$ thick disks, which were thinned to $100 \mu \mathrm{m}$ by grinding with abrasive paper. TEM samples in the shape of $3 \mathrm{~mm}$ disks were twin-jet polished with Struers A2 polishing solution at $17 \mathrm{~V}$ and $-20^{\circ} \mathrm{C}$. A JEOL $200 \mathrm{CX}$ transmission electron microscope (TEM) was used to investigate the microstructures of the low cycle fatigue specimens at an accelerating voltage of $200 \mathrm{KV}$.

\section{RESULTS}

The stress versus number of fatigue cycles (S-N) curves for $210 \mu \mathrm{m}$ grain samples is shown in Fig. 1(a). Those for the 70 $\mu \mathrm{m}$ counterparts are given in Fig. 1(b). Here, we observed in both cases initial softening if the strain amplitude is lower than $1.6 \%$, independent of the grain sizes.

At the same time, there is a re-entrant hardening following this initial softening if the strain amplitude falls between $0.4 \%$ and $0.8 \%$ for the $210 \mu \mathrm{m}$ samples (Fig. 1(a)) or within $0.2 \%$ and $0.4 \%$ (Fig. 1(b)) for the $70 \mu \mathrm{m}$ samples. This re-entrant hardening phenomenon diminished as the strain amplitude increasing, and eventually a hardening effect took place from the outset, as reflected in the S-N curves for strain amplitudes of $1.6-2 \%$ for both grain sizes. Comparing the S-N curves between Figs. 1(a) and (b), we see the initial hardening rates ( $2 \%$ strain amplitude) are comparable, although the re-entrant hardening rate for the $70 \mu \mathrm{m}$ sample is higher than that for the $210 \mu \mathrm{m}$ samples. In all, the initial and final response stresses are larger for the in $210 \mu \mathrm{m}$ samples for strain amplitudes range from $0.1 \%$ to $1.6 \%$ with the exception of two extreme amplitudes of $0.05 \%$ and $2 \%$.

The representative TEM micrographs taken from the $70 \mu \mathrm{m}$ samples, which were subjected to strain amplitudes of $0.05 \%$, $0.2 \%, 0.8 \%$ and $2 \%$, are shown in Figs. 2 (a)-(d), respectively. All samples demonstrate a cellular structure regardless of the strain amplitude, similar to the $210 \mu \mathrm{m}$ cases.

This seems to suggest that fatigued IF steel would exhibit a cellular structure regardless what the strain amplitudes or grain sizes are, there are indeed some subtle differences among the cellular structures in cellular sizes, shapes and dislocation

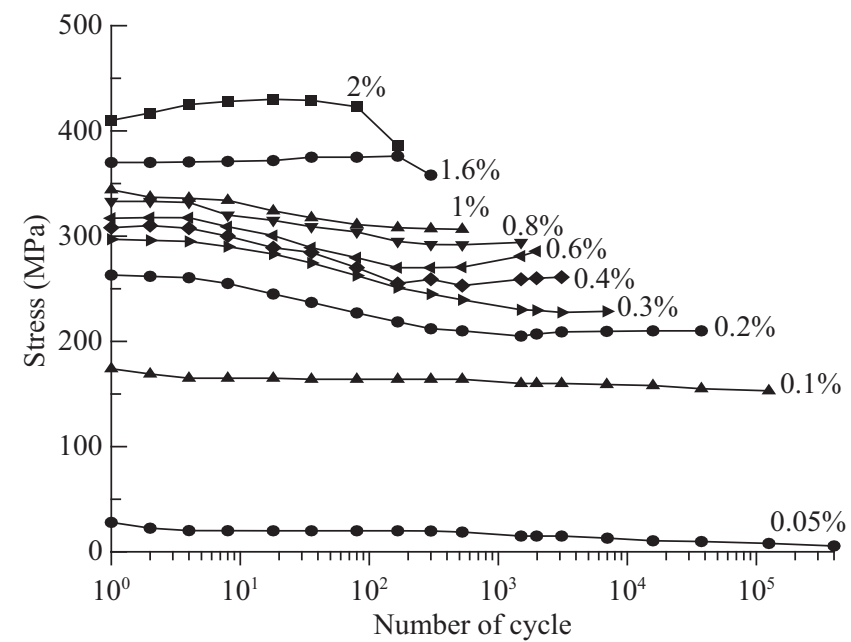

(a)

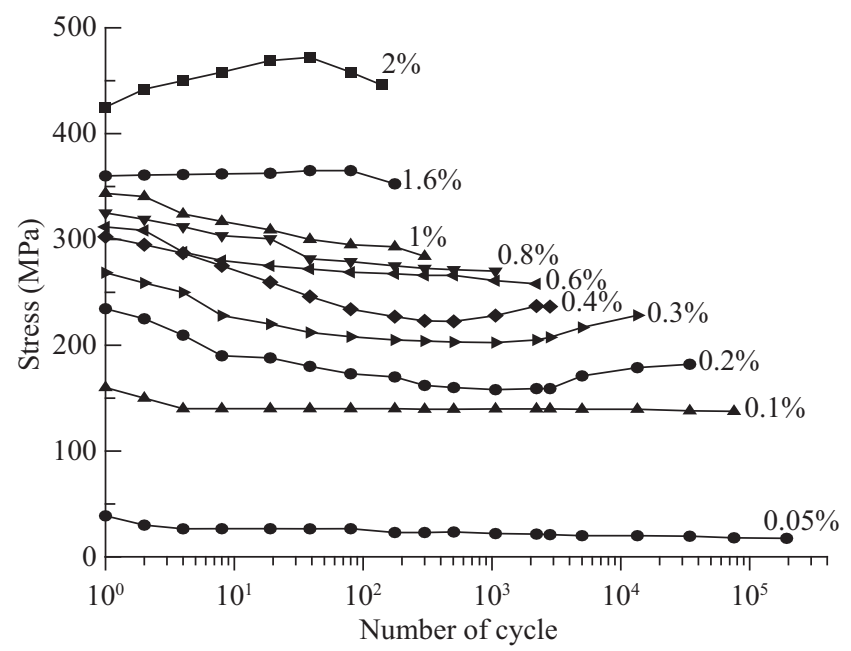

(b)

Fig. 1. The S-N curve in specimen was fatigue at various strain amplitudes: (a) $210 \mu \mathrm{m}$, (b) $70 \mu \mathrm{m}$.

densities, though. The dislocation evolution for $0.1 \%, 0.2 \%$ and $1 \%$ strain amplitudes is as follows.

Fig. 3(a) shows the uncondensed walls embedded in the dislocation matrix after 1000 fatigue cycles with exposure to $0.1 \%$ strain amplitude. Under the same strain amplitude, the uncondensed dislocation cellular structure grew larger after 10000 fatigue cycles (Fig. 3(b)). PSBs and small cells started to form in the larger dislocation cells after 40000 fatigue cycles (Fig. $3(\mathrm{c}))$. When the strain amplitude increased to $0.2 \%$, as indicated in Fig. 4(a), the PSBs in the larger dislocation cells started to emerge after 500 fatigue cycles. Smaller dislocation cells existing in larger cellular structures were found at an initial stage after 6000 cycles, as shown in Fig. 4(b). For the 1\% strain amplitude case, as shown in Fig. 5(a), the embedded larger uncondensed dislocation cells were observed after a mere 1 fatigue cycle. For completeness, Figs. 5(b) and (c) are archived herein, showing the cellular dislocation structural difference in sizes and densities after 100 and 300 fatigue cycles. 

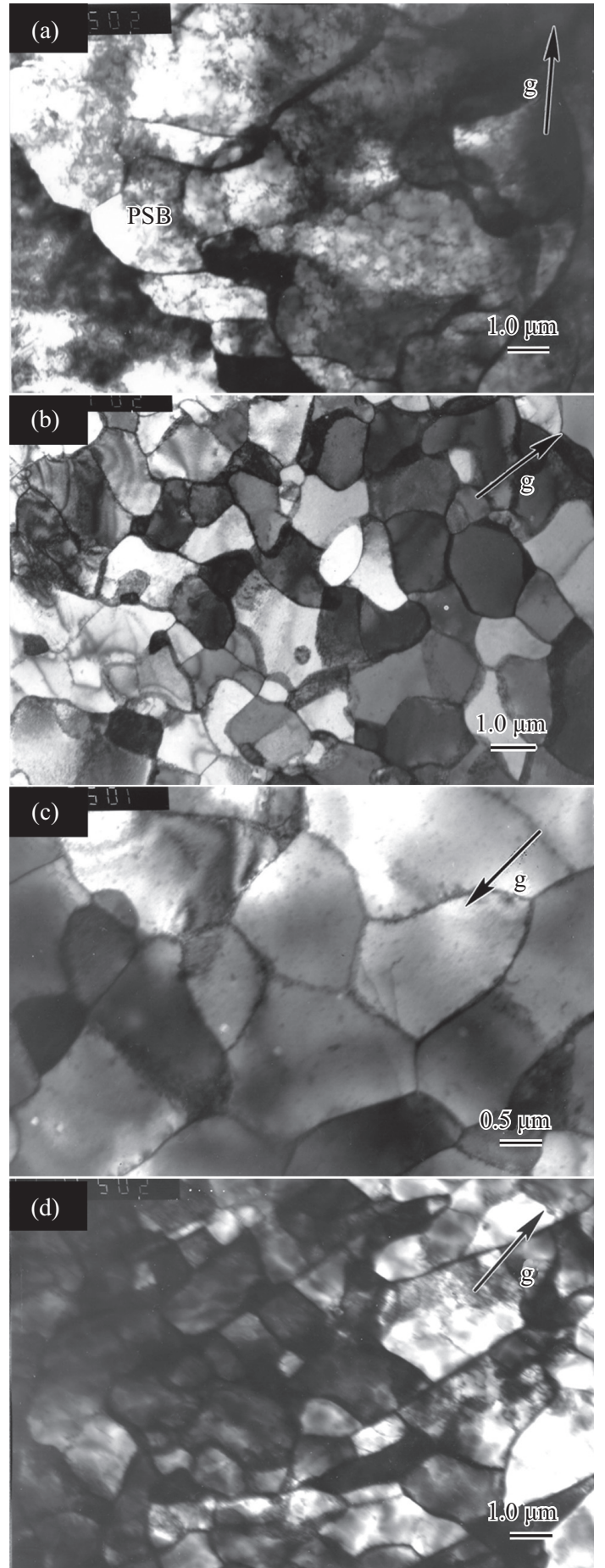

Fig. 2. The dislocation cell structure at various strain amplitude was observed in $70 \mu \mathrm{m}$ grain size specimen: (a) $0.05 \%$, (b) $0.2 \%$, (c) $0.8 \%$, (d) $2 \%$.

\section{DISCUSSION}

There was an initial softening effect, as presented above in Figs. 1(a) and (b), when the strain amplitude was lower than $1.6 \%$, because it is easier to activate the multiple slip systems in the BCC materials. As a result, once the dislocations started
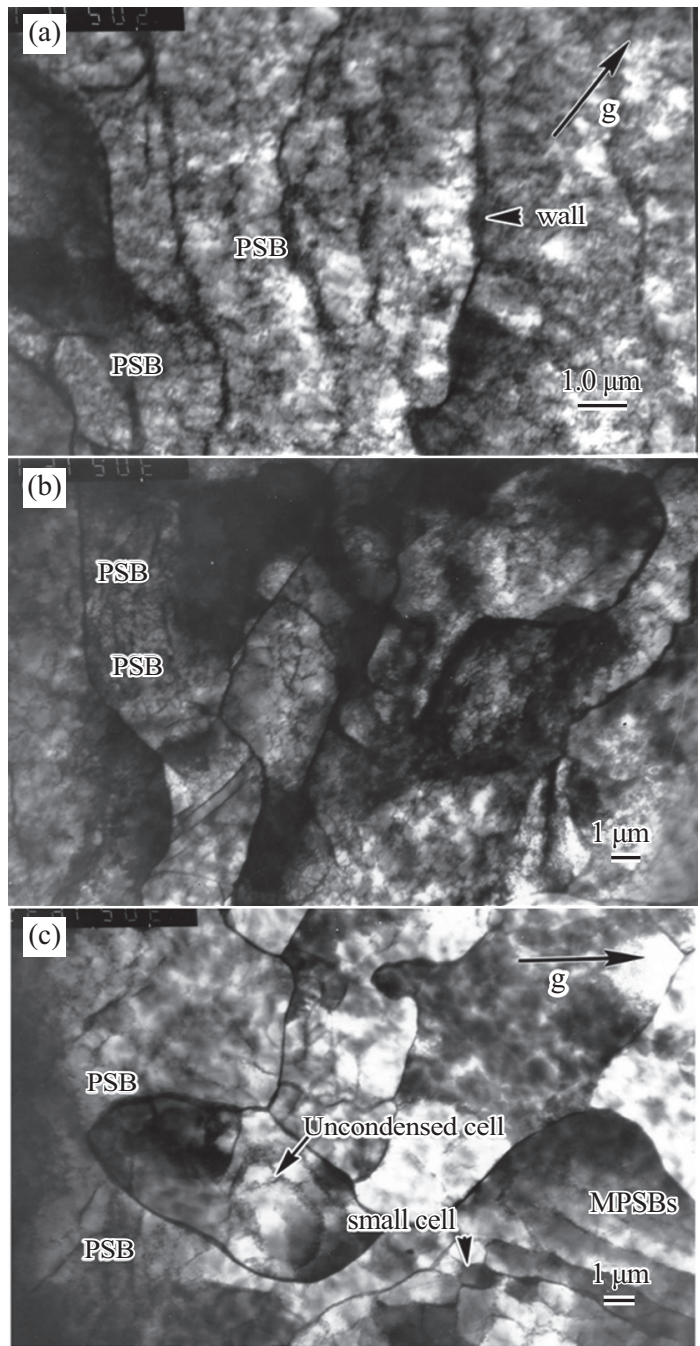

Fig. 3. The dislocation cell structure at $0.1 \%$ strain amplitude in $70 \mu \mathrm{m}$ grain size specimen was observed at (a) 1000 cycles, (b) 10000 cycles, and (c) 40000 cycles during fatigue.

to pile up, re-entrant hardening would take place under some circumstances. Here, as dislocations were generated and piled up at the early stage in the fatigue process, it would trigger a second slip system into the operation mode (Fig. 3(a)).

This accounts for the softening effect shown in the S-N curves. However, the initial hardening effect observed for a strain amplitude of $1.6 \%$ arises from the activation of more multiple slip systems to participate in the formation of cellular structures at the initial stage of the fatigue test (Fig 5). The unusual exceptions for the extreme cases of $0.05 \%$ and $2 \%$ strain amplitudes, for which the response stress of the $70 \mu \mathrm{m}$ samples is larger compared to the $210 \mu \mathrm{m}$ samples, is attributed to the strain localization at the grain boundaries.

Since the grain boundaries for the $70 \mu \mathrm{m}$ samples are larger, while at the same time the strain amplitude of $0.1 \%$ is too small to activate any multiple slip systems near the grain boundaries, the dislocations would pile up near the grain boundaries. It is thus understandable for the smaller grains to 


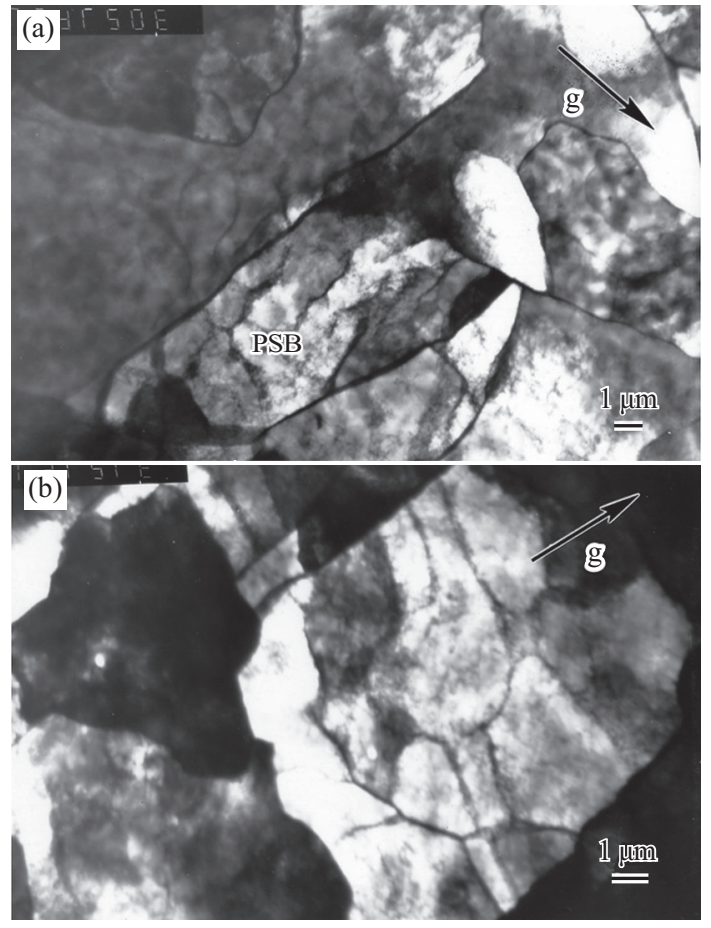

Fig. 4. The dislocation cell structure at $0.2 \%$ strain amplitude in $70 \mu \mathrm{m}$ grain size specimen was observed at (a) 500 cycles and (b) 6000 cycles during fatigue.

be more effective in blocking the movement of the dislocations, thus leading to a higher fatigue stress.

For the other extreme in the $2 \%$ case, the strain amplitude is so large that multiple slips would take place regardless of the grain sizes. However, this effect is more pronounced for the smaller grains, hence, in this case it shows higher fatigue stress, contrary to FCC structure materials, such as copper.

In typical cases show strain amplitudes between $0.1 \%$ and $1.6 \%$, with the response stresses in the large grain samples higher because the strain localization effect $[6,7]$ in smaller grains is larger because the multiple slip systems could be activated at an earlier stage. Note that the second slip systems are responsible for the softening effect in the fatigue processes [1]. In this case, the initial response stress in larger grains would be higher. However, this difference diminishes as the strain amplitude increases due to the smaller strain localization effect caused by larger strain amplitude.

The difference in response stress at fatigue also decreases as the fatigue cycles increases in spite of the strain amplitude, as long as it falls within $0.2 \%$ and $1.6 \%$. This results in the development of dislocations into other low energy forms as the fatigue cycles increase, regardless what the grain sizes are. Here, the strain localization effect $[6,7]$ has decreased with increasing fatigue cycles; hence, the response stresses are similar despite the difference in grain sizes at the fatigue test final stage.

As the re-entrant hardening effect commences, a large uncondensed cellular structure would start to form early on, and
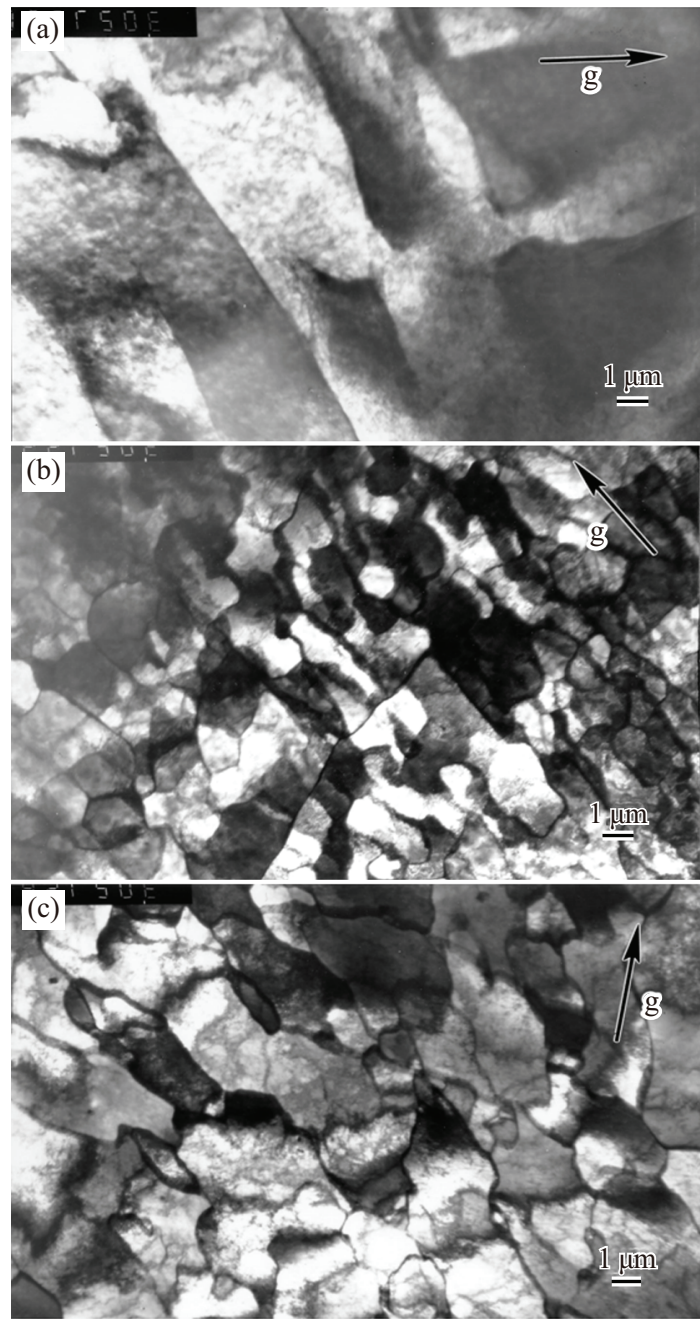

Fig. 5. The dislocation cell structure at $1 \%$ strain amplitude in $70 \mu \mathrm{m}$ grain size specimen was observed at (a) 1 cycle, (b) 100 cycles, and (c) 300 cycles during fatigue.

the multiple PSBs also began to develop into larger cells (Fig. 4(a)) as the fatigue cycles increase. This stage reflects the softening in stress as presented in the S-N curves, which is consistent with the dislocation structures associated with the PSBs reported previously by Llanes et al. [6, 7]. With increased fatigue cycles, the dislocations develop into a cellular structure at 6000 cycles (Fig. 4(b), $0.2 \%$ strain amplitude).

In the S-N curves, this stage corresponds to hardening, also in agreement with reference [1]. Based on the above findings, the re-entrant hardening should arise because of easier activation of the multiple slip systems for the BCC materials when the strain amplitude is too small to create small cells at initial stage. As a result, the multiple slip systems operated at low strain amplitude would induce large uncondensed cells to be embedded in a dislocation matrix (Figs. 3(a) and (b)).

Under this condition, the occupied space of the dislocation cells is large enough for the dislocation structure to continue to evolve within, leading to the formation of smaller dislocation cells, and, therefore, the re-entrant hardening. 
The sizes of the dislocation cells largely decrease with increasing strain amplitude for FCC materials. However at $0.2 \%$ strain amplitude, the dislocation cell size (Fig. 2(b)) is smaller than those for the $0.8 \%$ cases (Fig. 2(c)). In BCC materials, it is easier to activate the multiple slip system regardless of the strain amplitude. The differences concerning the multiple slip systems between the high and low strain amplitude cases are mainly in the number of slip systems as well as their operation rates, which are higher at higher strain amplitudes, leading to faster formation of smaller cells for large strains (Figs. 2(d), 5(b) and 5(c)) and larger cells for low strain amplitudes (Fig. 2(a)).

However, these large cells would form into uncondensed structures (Figs. 3(a), 3(b) and 4(a)), and then evolve into low energy condensed cell structures with increasing fatigue cycles (Figs. 2(b), 3(c) and 4(b)). Based on the results in Fig. 3, the uncondensed walls become embedded into the dislocation matrix (Fig. 3(a), at 1000 cycles) at first, but then evolve into uncondensed cellular structures (Fig. 3(b)) as the fatigue cycles reaches 10000. The multiple slip systems (PSBs) (Figs. $3(c)$ and 4(a)) emerged in the dislocation matrix located in the larger cells with increased fatigue cycles, eventually forming a small sized cellular structure (Figs. 3(c) and 4(b)) in larger cells that were created at an earlier stage.

In summary, smaller cellular structure may evolve from a large uncondensed cellular structure because the size of cells is so large that multiple slip systems are activated into operation in the larger cells. Therefore, the dislocation cell size at $0.2 \%$ strain amplitude is smaller than at $0.3 \%$ and $0.8 \%$ strain amplitudes.

\section{CONCLUSIONS}

1. There is an initial softening if the strain amplitude is lower than $1.6 \%$. Under such circumstances, the cellular form microstructure forms independent of the strain amplitude and grain size in BCC IF steel. This is because of the easier activation of multiple slip systems.

2. A re-entrant hardening effect was observed for strain amplitudes range from $0.2 \%$ to $0.4 \%$ in samples of $70 \mu \mathrm{m}$ grains and from $0.4 \%$ to $0.8 \%$ for the $210 \mu \mathrm{m}$ samples. Small cellular structures formed within larger uncondensed cells at an initial stage.

3. The size of dislocation cells obtained from the test with $0.2 \%$ amplitude was smaller compared with those from the $0.8 \%$ amplitude because of the larger cellular size associated with low strain amplitude. Consequently, the occupied space of larger cells becomes large enough to induce dislocation evo- lution and form smaller cells (PSBs) in the larger cells.

4. The fatigue behavior is largely similar for both grains sizes, with subtle differences determined by the strain localization effect and the rate of activating multiple slip systems into operation.

5. The fatigue saturation stress at $0.05 \%$ and $2 \%$ strain amplitudes in the $70 \mu \mathrm{m}$ samples is larger in comparison with those of the $210 \mu \mathrm{m}$ samples, dissimilar to materials of FCC structure.

\section{REFERENCES}

1. Ackermann, F., Kubin, L. P., Lepinoux, J., and Mughrabi, H., "The dependence of dislocation microstructure on plastic strain amplitude in cyclically strained copper single crystals," Acta Metallurgica, Vol. 32, No. 5 , pp. 715-725 (1984).

2. Huang, H. L. and Ho, N. J., "The microstructure of the fatigue crack tip in Fe-Al-Mn- $0.4 \% \mathrm{C}$ alloy near the stress intensity threshold," Materials Science \& Engineering A, Vol. 293, pp. 235-241 (2000).

3. Kaneshiro, H., Katagiri, K., Makabe, C., Yafuso, T., and Kobayashi, H., "Dislocation structures in the strain localized region in fatigued 70/30 brass and the interaction with grain boundary," Metallurgical and Materials Transactions A, Vol. 21, No. 3, pp. 667-671 (1990).

4. Li, Y. and Laird, C., "Cyclic response and dislocation structures of AISI 316L stainless steel. part 1: single crystals fatigued at intermediate strain amplitude," Materials Science \& Engineering A, Vol. 186, pp. 65-86 (1994).

5. Li, Y. and Laird, C., "Cyclic response and dislocation structures of AISI 316L stainless steel. part 2: polycrystals fatigued at intermediate strain amplitude," Materials Science \& Engineering A, Vol. 186, pp. 87-103 (1994).

6. Llanes, L. and Laird, C., "The role of annealing twin boundaries in the cyclic deformation of F.C.C. materials," Materials Science \& Engineering $A$, Vol. 157, pp. 21-27 (1992).

7. Llanes, L., Rollett, A. D., Laird, C., and Bassani, J. L., "Effect of grain size and annealing texture on the cyclic response and the substructure evolution of polycrystalline copper," Acta Metallurgica et Materialia, Vol 41, No. 9, pp. 2667-2679 (1993).

8. Mughrabi, H., Herz, K., and Stark, X., "Cyclic deformation and fatigue behaviour of $\alpha$-iron mono-and polycrystals," International Journal of Fracture, Vol. 17, No. 2, pp. 193-220 (1981).

9. Mughrabi, H., Herz, K., and Stark, X., "The effect of strain-rate on the cyclic deformation properties of $\alpha$-iron single crystals," Acta Metallurgica, Vol. 24, No. 7, pp. 659-668 (1976).

10. Nine, H. D., "Slip planes and asymmetric slip in fatigue of Fe single crystals," Philosophical Magazine. Vol. 26, No. 6, pp. 1409-1418 (1972).

11. Raouf, H. A. and Plumtree, A., "The influence of deformation rate on the cyclic behavior of pure iron," Metallurgical and Materials Transactions $B$, Vol. 2, pp. 1863-1867 (1971).

12. Winter, A. T., Pedersen, O. B., and Rasmussen, K. V., "Dislocation microstructures in fatigued copper polycrystals," Acta Metallurgica, Vol. 29 , No. 5, pp. 735-748 (1981). 